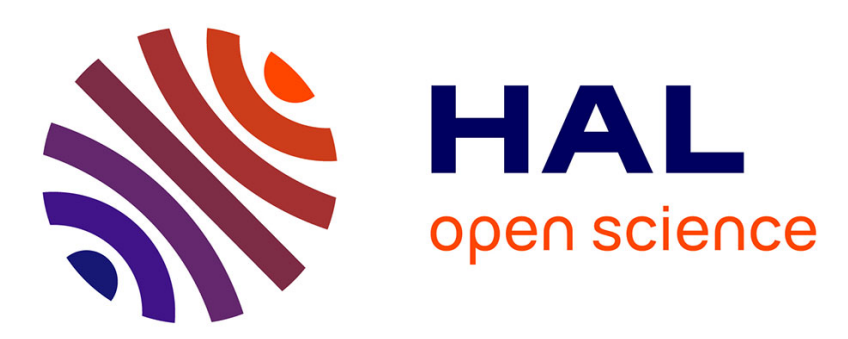

\title{
A unified probabilistic model of the perception of three-dimensionnal structure from optic flow
}

Francis Colas, Jacques Droulez, Mark Wexler, Pierre Bessiere

\section{To cite this version:}

Francis Colas, Jacques Droulez, Mark Wexler, Pierre Bessiere. A unified probabilistic model of the perception of three-dimensionnal structure from optic flow. 2006. inria-00182001

\section{HAL Id: inria-00182001 \\ https://hal.inria.fr/inria-00182001}

Submitted on 24 Oct 2007

HAL is a multi-disciplinary open access archive for the deposit and dissemination of scientific research documents, whether they are published or not. The documents may come from teaching and research institutions in France or abroad, or from public or private research centers.
L'archive ouverte pluridisciplinaire $\mathbf{H A L}$, est destinée au dépôt et à la diffusion de documents scientifiques de niveau recherche, publiés ou non, émanant des établissements d'enseignement et de recherche français ou étrangers, des laboratoires publics ou privés. 


\title{
A unified probabilistic model of the perception of three-dimensional structure from optic flow
}

\author{
Francis Colas ${ }^{1}$, Jacques Droulez $^{2}$, Mark Wexler $^{2}$, Pierre Bessière $^{3}$ \\ 1 INRIA-Gravir laboratory, INRIA Rhônes-Alpes, 655 avenue de l'Europe, 38334 Montbonnot, France \\ 2 Laboratoire de la Physiologie de la Perception et de l'Action, Collège de France, 11 place Marcellin Berthelot, 75005 Paris, \\ France \\ ${ }^{3}$ CNRS-Gravir laboratory, INRIA Rhônes-Alpes, 655 avenue de l'Europe, 38334 Montbonnot, France
}

The date of receipt and acceptance will be inserted by the editor

\begin{abstract}
Human observers can perceive the threedimensional (3-D) structure of their environment using various cues, an important one of which is motion parallax. The motion of any point's projection on the retina depends both on the point's movement in space and on its distance from the eye. Therefore, retinal motion can be used to extract the 3-D structure of the environment and the shape of objects, in a process known as structurefrom-motion (SFM). However, because many combinations of 3-D structure and motion can lead to the same optic flow, SFM is an ill-posed inverse problem. The rigidity assumption is a constraint supposed to formally solve the SFM problem and to account for human performance. Recently, however, a number of psychophysical results, in both moving and stationary human observers, have shown that the rigidity assumption alone cannot account for SFM, but no model is known to account for the new results. Here, we construct a Bayesian model of SFM based on only one new assumption, that of stationarity, coupled with the assumption of rigidity. The predictions of the model, calculated using a new and powerful methodology called Bayesian programming, account for a wide variety of experimental findings.
\end{abstract}

\section{Introduction}

Relative motion between an observer and the objects in a visual scene leads to a deformation of the image on the retina, called optic flow. Optic flow depends heavily on the $3-\mathrm{D}$ shapes and motions of the objects in a scene, and can therefore be used to extract 3-D information about scene geometry. The capacity to extract 3-D shape or structure from motion (SFM), also known as motion parallax or the kinetic depth effect, was noticed by von Helmholtz (1867) and was first experimentally quantified by Wallach and O'Connell (1953).
Although it is simple to derive the optic flow corresponding to given 3-D geometry and motion, perception faces the inverse problem, to derive $3-\mathrm{D}$ shape and motion from optic flow. Because an infinite number of combinations of geometry and motion can lead to the same optic flow, SFM is an ill-posed inverse problem.

It is commonly believed that the SFM problem is at least partly solved by a constraint called the rigidity assumption, the hypothesis that optic flow is due to $3-\mathrm{D}$ translations and rotations of a rigid body. This drastically reduces the number of degrees of freedom associated with motion, and it can be shown that under this assumption, both structure and motion can theoretically be recovered from very little optic flow information (Ullman 1979). Several algorithms based on the rigidity assumption for special cases, such as planes, have been developed (Mayhew and Longuet-Higgins 1982). Psychophysical results show that human performance on some SFM tasks is at least broadly consistent with predictions based on the rigidity assumption (Wallach and O'Connell 1953; Koenderik 1986). More recent affine models are based only on local velocity information, rather than on the entire optic flow field, to account for human perception (Todd and Bressan 1990; Todd and Norman 1991).

Most studies of SFM involve an immobile observer experiencing optic flow consistent with moving 3-D objects. However, it is known that SFM is also effective when optic flow is generated by the observer's own head movement about a stationary 3-D scene (Rogers and Graham 1979). Until recently, it has been thought that 3D shapes perceived in subject-motion SFM are the same as those perceived in object-motion SFM, as long as the optic flow is the same (Wallach et al. 1974; Rogers and Graham 1979). However, in some cases, this turns out to be false: even when optic flow is kept constant, the observer's movement influences perceived 3-D shape (Rogers and Rogers 1992; Dijkstra et al. 1995; Wexler et al. 2001b). 
The way in which self-motion influences perceived 3D shape leads us to postulate a second assumption in the interpretation of optic flow, that of stationarity: the visual system prefers the solution whose motion is minimal in an observer-independent, allocentric reference frame (Wexler et al. 2001b,a; Wexler 2003). While the rigidity assumption may be seen as the minimization of relative motion between the points of a possible object, the stationarity assumption is the minimization of absolute motion in an observer-independent reference frame. Taken separately, neither the stationarity nor the rigidity assumption can explain human SFM performance. However, until now, no coherent model has integrated these two assumptions.

In this article, we present a generic Bayesian model that integrates the stationarity and rigidity assumptions in the perception of 3-D planar surfaces from optic flow. The model not only accounts for SFM performance in moving and stationary observers that led to the postulation of the stationarity assumption, but also for a number of other, sometimes puzzling, results that have been previously reported. We investigate experiments focusing on the monocular perception of a rotating planar patch with a neutral or non-informative texture. In these experiments, motion was the only cue for plane orientation. Various variations of this common setup involve the motion of the observer's head or eyes, or the plane, or both, as well as the size of the displayed stimulus. Although perception of planes is a special case, it is a very important special case of spatial vision, as the visual world is composed mostly of surfaces, which, if sufficiently regular, can be locally approximated as planes.

In recent years, growing attention has been paid to Bayesian inference, as a common theoretical framework, to understand perceptive skills and multimodal interactions (Weiss et al. 2002; Ernst and Banks 2002; Kersten et al. 2004). In most works, however, the probabilistic reasoning has been limited to simplified forms of Bayes' theorem. Simplification either resulted from the use of the linear Gaussian assumption, which is not valid in the case of optic flow processing, or because inference was limited to a combination of prior knowledge and a set of observations. In order to combine several hypotheses, such as rigidity and stationarity, in a mathematically correct form, we found it necessary to put perception models back into a more general Bayesian framework that includes not only observed sensory data and perceived states, but also intermediate variables. Focusing on the SFM problem, we show here that our general Bayesian formalism allows us to express and to test several hypotheses originating from psychophysical experiments, in a very natural and efficient way.

\section{Methods}

We first present a generic, unified model of perception of the structure of an object from optic flow. Then, we give a precise instantiation for the perception of planes that yields the results presented in section 3 .

\subsection{Generic model}

The generic Bayesian model we propose is the expression of the hypotheses evoked above. The first two are the stationarity ( $\mathrm{H} 1)$ and rigidity $(\mathrm{H} 2)$ assumptions. We also assume that the structure of the object is independent of its motion, the motion of the observer and the conditions of observation (H3), and that the conditions of observations are independent of the motions of both the object and the observer (H4). We follow the Bayesian programming framework to specify a model with these hypotheses (Lebeltel et al. 2004). This model uses probabilities to represent and handle the uncertainty faced by an observer. This is a model of what an observer can deduce from the limited information of optic flow.

From relevant information to variables The unified model is based on relevant variables common to all instances of structure-from-motion perception. Additional variables can be used to comply with specific experimental conditions.

In this context, we propose a model that takes into account: (i) the observed optic flow (noted $\boldsymbol{\Phi}$ ), (ii) the 3-D structure of the object (noted $\Theta$ ), (iii) the motion of the object (noted $X$ ) in the observer's reference frame, (iv) the motion of the observer in the allocentric reference frame (noted $\mathbf{M}$ ), and ( $\mathrm{v}$ ) the general viewing conditions as defined by the experimental protocol (noted A).

From dependencies to decomposition At the core of a Bayesian model lies the joint probability distribution over all its variables. This joint distribution is the expression of the hypotheses of a model. The structural part in the specification of the joint distribution summarizes the dependencies and independencies between the variables. This structure is called decomposition.

Hypothesis $\mathrm{H} 1$ is the rigidity assumption, which states that the observed optic flow is most likely that of a rigid object. As a consequence, the optic flow depends on the relative motion, the structure of the object and the viewing conditions but is independent of self-motion. This corresponds to the following mathematical simplification:

$$
P(\boldsymbol{\Phi} \mid \Theta \mathbf{M} X \Lambda)=P(\boldsymbol{\Phi} \mid \Theta X \Lambda)
$$

The stationarity assumption (H2) states that object motion is most likely to be small in the allocentric reference frame. Therefore, the relative motion depends on self-motion. We use Bayes' rule to write:

$$
P(\mathbf{M} X)=P(\mathbf{M}) P(X \mid \mathbf{M}) \text {. }
$$


Hypothesis H3 states that the structure of the object is independent of the relative motion of the object, the self-motion, and the conditions of observation. This translates as a product of independent factors in the decomposition:

$$
P(\Theta \mathbf{M} X \Lambda)=P(\Theta) P(\mathbf{M} X \Lambda) .
$$

The last assumption (H4) states the independence between the motions and the general viewing conditions:

$$
P(\mathbf{M} X \Lambda)=P(\mathbf{M} X) P(\Lambda) .
$$

Finally, using Bayes' rule, we can write :

$$
P(\Theta \mathbf{M} X \Lambda \mathbf{\Phi})=P(\Theta \mathbf{M} X \Lambda) P(\boldsymbol{\Phi} \mid \Theta \mathbf{M} X \Lambda) .
$$

Putting together equations 5, 3, 4, 2, and 1, we obtain the decomposition, shown in equation 6 , that is the structural expression of our hypotheses.

$$
\begin{aligned}
P(\Theta \mathbf{M} X \Lambda \mathbf{\Phi})= & P(\Theta) P(\Lambda) P(\mathbf{M}) \\
& \times P(X \mid \mathbf{M}) \\
& \times P(\mathbf{\Phi} \mid \Theta X \Lambda) .
\end{aligned}
$$

From physical and physiological laws to distributions In order to get a usable expression for the joint distribution, we must specify each of the five factors of the above decomposition.

The first factor, $P(\Theta)$, is the prior on the structure of the object. It represents what the model of an observer expects before any observation. It can be an uninformative prior or it can reflect some bias in perception, in favor of more common shapes.

In the same way, $P(\mathbf{M})$ and $P(\Lambda)$ represent respectively the expectation by an observer of her or his own motion, and of the conditions of observation. If we consider that the model has an exact knowledge of them (as will be the case later in this article), this probability distribution is simplified in the final inference and thus can be left unspecified.

The fourth factor $P(X \mid \mathbf{M})$ specifies the relative motion expected from a given self-motion. According to stationarity, the object is more likely to undergo a smaller absolute motion. Therefore, the most probable relative motion should be defined as the opposite of self-motion. The actual parametrical form varies once again with the experiment, but a general expression could be proportional to the exponential of the opposite of kinetic energy (Gibbs distribution). In some cases, this means a Gaussian distribution.

The last factor in decomposition 6 is the distribution of optic flow, given the structure of the object, the relative motion between the object and the observer, and the conditions of observation, $P(\boldsymbol{\Phi} \mid \Theta X \Lambda)$. Following the rigidity assumption, this distribution can express that the most probable optic flow is the theoretical flow of the object in this particular configuration, given this particular motion.
Formalized questions A probabilistic question is the distribution over some variables of the model, possibly given the knowledge of the values of other variables. With a completely specified joint distribution, the answers to such questions can be mechanically inferred with the rules of probability calculus.

The SFM question is the probability of the object structure or shape, given the optic flow, the self-motion, and the general conditions of observation written as $P(\Theta \mid \boldsymbol{\phi} \mathbf{m} \lambda) .{ }^{1}$ This question is answered by the following expression, given by Bayesian inference:

$$
\begin{aligned}
& P(\Theta \mid \boldsymbol{\phi} \mathbf{m} \lambda) \\
\propto & P(\Theta) \sum_{x \in X} P(x \mid \mathbf{m}) P(\boldsymbol{\phi} \mid \Theta x \lambda) .
\end{aligned}
$$

This is essentially the problem we solved to obtain the results shown later in this article. Given observations of optic flow and self-motion, this distribution represents knowledge about the structure of the object (including its relative position with respect to the observer) that one can infer from our hypotheses. This is what is often referred to as a statistically optimal observer, given these two assumptions.

Furthermore, the same probabilistic model can be used to answer other questions. For example, one may be interested in the estimation of self-motion from optic flow: $P(\mathbf{M} \mid \phi \lambda)$. This question can be used to study vection, where optic flow induces the sensation of selfmotion, and the direction of perceived self-motion, called heading. For this question, Bayesian inference with the same model gives the following expression:

$$
\begin{aligned}
& P(\mathbf{M} \mid \boldsymbol{\phi} \lambda) \\
\propto & P(\mathbf{M}) \sum_{x \in X, \theta \in \Theta} P(\theta) P(x \mid \mathbf{M}) P(\boldsymbol{\phi} \mid \theta x \lambda) .
\end{aligned}
$$

\subsection{The case of a moving dotted plane}

The generic model is a template, which must be adapted to account for particular experiments. Here we present the exact instantiated model used to generate the results presented below, for the perception of a moving planar object.

Variables The structure $\Theta$ of the object is reduced to the position and orientation of the plane. As one point of the plane is already known (the fixation point) ${ }^{2}$, only two orientation parameters are needed to parametrize the structure of the object. For practical reasons, we use the depth gradients along the transversal and vertical axes. If we call $x, y$, and $z$ the coordinates of a point of the plane along the transversal, vertical, and sagittal axes

\footnotetext{
1 We use an uppercase letter for a variable and lowercase for the instantiation of a variable with a particular value.

2 By convention the distance between the fixation point and the observer is taken as the unit of distance. This way the scale issue disappears.
} 
respectively, then the structure $\Theta$ is the pair $(\chi, v)=$ $\left(\frac{\partial z}{\partial x}, \frac{\partial z}{\partial y}\right)$.

Self-motion $\mathbf{M}$ is a set of translation and rotation velocities of the observer, chosen along the transversal, vertical, and sagittal axes. Likewise, relative motion is decomposed into its rotation and translation components, $\boldsymbol{\Omega}$ and $\mathbf{T}$ respectively.

In the case of planar objects, the optic flow is entirely specified by eight components (see appendix for details), namely the two velocity components at the origin $\left(\boldsymbol{\Phi}^{0}\right)$, the four first-order derivatives of the velocity field at the origin $\left(\boldsymbol{\Phi}^{1}\right)$, and the two independent components of the second-order derivatives of the velocity field at the origin $\left(\boldsymbol{\Phi}^{2}\right)$ (Longuet-Higgins 1984).

Finally, we restrain the viewing condition parameters to the most critical one, the size of the field of view.

Distributions The prior on plane orientation $P(\Theta)$ is chosen to be the least informative, so as not to bias the inference. This corresponds to a prior invariant to arbitrary rotation of the plane.

For sFm question $P(\Theta \mid \boldsymbol{\phi} \mathbf{m} \lambda)$, both self-motion, $\mathbf{m}$, and the size of field, $\lambda$, are known. The posterior distribution does not depend on the priors on variables $\mathbf{M}$ and $\Lambda$; therefore, these prior distributions do not need to be specified, as can be seen in expression 7 .

As for the expression of stationarity, the distribution of relative motion given self-motion yields the most probable relative motion as equal-and-opposite to selfmotion, corresponding to no absolute motion. To this end, we chose a Gaussian distribution centered on such relative motion. The Gaussian is the least informative distribution, given the mean and the uncertainty of the distribution. It also corresponds to the Gibbs distribution with kinetic energy.

Likewise, the distribution of optic flow, given the relative motion and orientation of the plane and the size of the field of view, is an expression of the rigidity assumption. We chose a Gaussian distribution centered on the theoretical values of the eight components (see expression in appendix A). The field of view is assumed to change the variance of the second-order components $\left(\boldsymbol{\Phi}^{2}\right)$.

Implementation Although the specified distributions are either Gaussian or uniform, the SFM question has no analytical solution because of the intrinsic nonlinearities of the optic flow equations (see appendix). Quantitative simulations are then performed by computing the exact inference on discretized variables. Table 1 gives the details of the ranges (minimum, maximum and number of samples in between) and dimensionality of each component of $\Theta$ (top row), of the relative rotation (second row), of the relative translation (third row), and of the size of the field of view (bottom row). Other variables do not need to be discretized as their values are known for the inference.
On the other hand, some of the distributions in our decomposition involve parameters. This is the case with the Gaussians on relative motion and optic flow. Covariance matrices of Gaussian distributions are defined in table 2. The variance of $\boldsymbol{\Phi}^{2}$ is larger in small field of vision to account for the inaccurate evaluation of second-order derivatives.

\begin{tabular}{|c|}
\hline Distribution parameters \\
\hline$\sigma_{\mathbf{T}}=0.3 * I d_{3 \times 3}$ in m.s $^{-1}$ \\
\hline$\sigma_{\Omega}=1.2 * I d_{3 \times 3}$ in rad.s \\
\hline$\sigma_{\Phi^{0}}=1.0 * I d_{2 \times 2}$ in $m . s^{-1}$ \\
\hline$\sigma_{\Phi^{1}}=0.025 * I d_{4 \times 4}$ in $s^{-1}$ \\
\hline$\sigma_{\Phi^{2}} \mid \lambda=S F$ \\
\hline$\sigma_{\Phi^{2}} \mid \lambda=L F$ \\
\hline
\end{tabular}

Table 2: Covariance matrices of each factor of the joint distribution. From top to bottom: distribution over the relative translation, relative rotation, order 0 optic flow, order 1 optic flow, order 2 optic flow in a small field of vision and order 2 optic flow in a large field of vision.

The results presented in the following section were all computed with this single set of parameters using the ProBT inference engine for the calculations (Lebeltel et al. 2004).

\section{Results}

There are numerous sources of ambiguity in the perception of optic flow. Figures 1 and 6 show five kinds of situations of motion of the object or the observer that generate approximately the same optic flow. They have been studied in detail by six sets of psychophysics experiments previously reported. We show that the Bayesian model compares to human performance in various conditions of motion of the plane, voluntary motion of the observer, and size of field of vision.

\subsection{Depth reversal}

Depth reversal is a well-known effect in three-dimensional vision: many depth cues are ambiguous about the sign of relative depth (cf. the Necker cube). In SFM the simplest instance of this ambiguity is the observation of a rotating plane through a small opening. In this case, there is an ambiguity on the tilt and direction of rotation, as illustrated in figure 1(b). The extrinsic orientation of a plane in 3-D space is often parametrized by two angles; slant and tilt. Slant is the angle, in 3-D space, between the plane's normal vector and the normal of the fronto-parallel plane. Tilt is the angle, in the frontoparallel plane, of the projection of the plane's normal. However it has been shown (Dijkstra et al. 1995) that this ambiguity does not hold for a large field of vision. 


\begin{tabular}{|c|c|c|c|c|c|}
\hline variable & symbol & $\min$ & $\max$ & number of values by dimmension & dimension \\
\hline Depth gradient & $\Theta$ & -4.125 & 4.125 & 33 & 2 \\
\hline Angular velocity & $\Omega$ & $-1.375{\mathrm{rad} . \mathrm{s}^{-1}}^{-1}$ & $1.375 \mathrm{rad} . \mathrm{s}^{-1}$ & 11 & 3 \\
\hline Linear velocity & $T$ & $-1.375 \mathrm{m.s}$ & $1.375 \mathrm{m.s}$ & 11 & 3 \\
\hline Size of field & $\Lambda$ & $0.015 \mathrm{sr}$ & $1.05 \mathrm{sr}$ & 2 & 1 \\
\hline
\end{tabular}

Table 1: Domains of the variables.

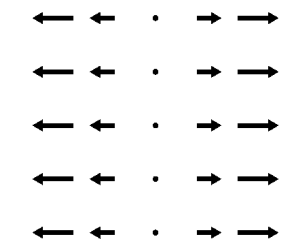

(a)
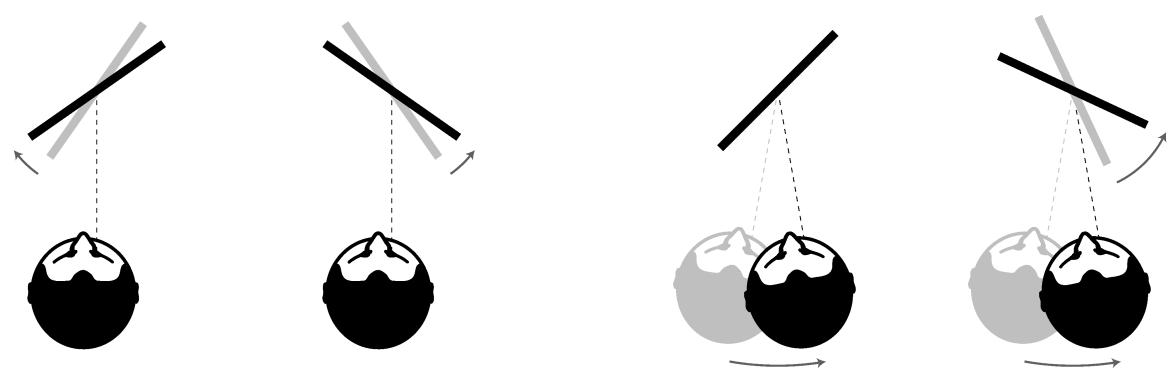

(b)

(c)
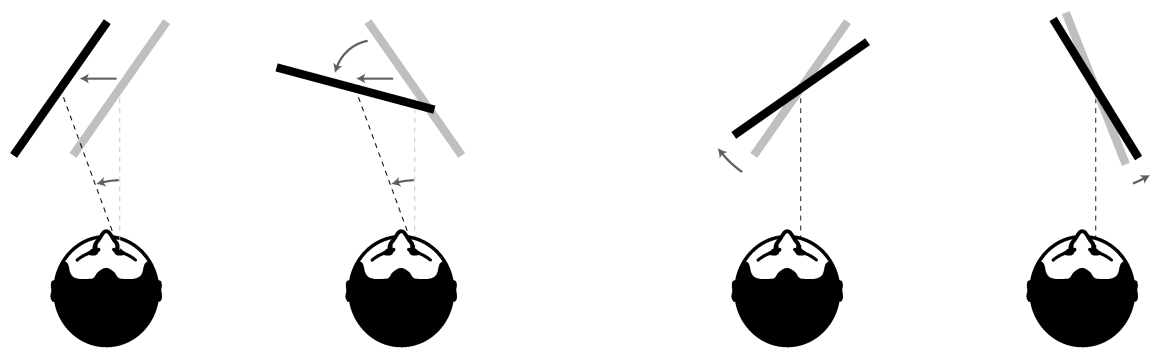

(d)

(e)

Fig. 1: Some ambiguities in first-order optic flow that have been used in the studies cited. (a) An example of an optic flow field that presents a number of ambiguities: all configurations shown in this figure lead to this flow. (b) The two configurations, which differ by simultaneous reversals of relative depth and 3-D motion, both yield the optic flow shown in (a). This ambiguity is called depth reversal. (c) Depth reversals can also occur for moving observers. The two configurations have the same relative motion between object and observer as in (b), and therefore yield the same optic flow. However, one solution is stationary in an allocentric or observer-independent reference frame, while the other solution undergoes a rotation in this frame, twice as fast as the observer's motion. (d) The same ambiguity when the observer tracks a moving surface with the eyes. One solution undergoes a translation only, while the other undergoes the same translation but also a rotation. (e) Ambiguity between slant and rotation speed: a larger slant coupled with a slower rotation speed may give the same optic flow as a lower slant together with a faster rotation.

We will investigate this simple effect as the first example of our model.

The experiment we use as a reference has been described by Cornilleau-Pérès et al. (2002). In this experiment, the stationary participant observes a planar patch in rotation about a fronto-parallel axis (the plane is painted with a uniform random dot texture). After the presentation of the stimulus, the observer is asked to estimate the orientation of the planar patch by aligning a probe to it. Two field-of-view sizes were compared: a large field with a $60^{\circ}$ aperture angle and a small field with an $8^{\circ}$ aperture angle.

Cornilleau-Pérès et al. (2002) report the results in terms of the rate of tilt reversals. A tilt reversal is defined to occur when absolute error in the estimation of the tilt angle is greater than $90^{\circ}$. The reversal rate can 


\begin{tabular}{|c|r|r|}
\hline Condition & Experiment & Model \\
\hline Small field & $48.8 \%$ & $44.6 \%$ \\
\hline Large field & $3.1 \%$ & $3.3 \%$ \\
\hline
\end{tabular}

Table 3: Influence of the size of field of vision on reversal rate. Both the experiments (Cornilleau-Pérès et al. 2002) and the Bayesian model exhibit less reversal percept in a large field of vision.

be considered a measure of the ambiguity, as illustrated in figure 1(b). The middle column of table 3 presents the results of the experiment, and we observe that the reversal rate drops from close to its maximal value $(50 \%)$ in small field of vision to below $5 \%$ in large field of vision.

Our Bayesian model computes the probability distribution over the orientation $\Theta$ of the plane, given the optic flow, the field of view and the observer's movement (example in figure 2). Ambiguity in the optic flow interpretation, such as illustrated in figure 1, results in a multimodal probability distribution. To compare the reversal rate reported by Cornilleau-Pérès et al. (2002) with model output, we computed the sum of probabilities corresponding to tilt errors greater than $90^{\circ}$ (see table 3 ).

This result is accounted for by the rigidity assumption. In our model, this assumption is expressed by a probability distribution over the optic flow (see Methods for details). The tilt ambiguity is a consequence of the invariance of the first-order components of the optic flow $\left(\boldsymbol{\Phi}^{1}\right)$ with respect to tilt reversal; therefore only the second-order components can disambiguate the stimulus.

In the Bayesian model, the standard deviation over the second-order optic flow is smaller in a large field than in a small field of vision. Therefore the influence of second-order optic flow is greater in a large field of vision than in a small field.

Qualitatively, insofar as this uncertainty is greater in a small field, the probability of reversal will always be higher in a small field than in a large field. Figure 3 shows the quantitative evolution of the reversal rate in the model as a function of this parameter.

\subsection{Depth reversals in moving and immobile observers}

Self-motion has been shown to modify depth perception from optic flow. This can be seen most clearly in studies that find differences in SFM performance in moving and immobile observers, while keeping optic flow the same in the two self-motion conditions. Thus, actively generated optic flow can lead to a different perception of 3-D shape than the same optic flow viewed passively by an immobile observer.

One of the ways in which self-motion modifies SFM is by diminishing the ambiguity that leads to depth reversals (Rogers and Rogers 1992; Dijkstra et al. 1995;

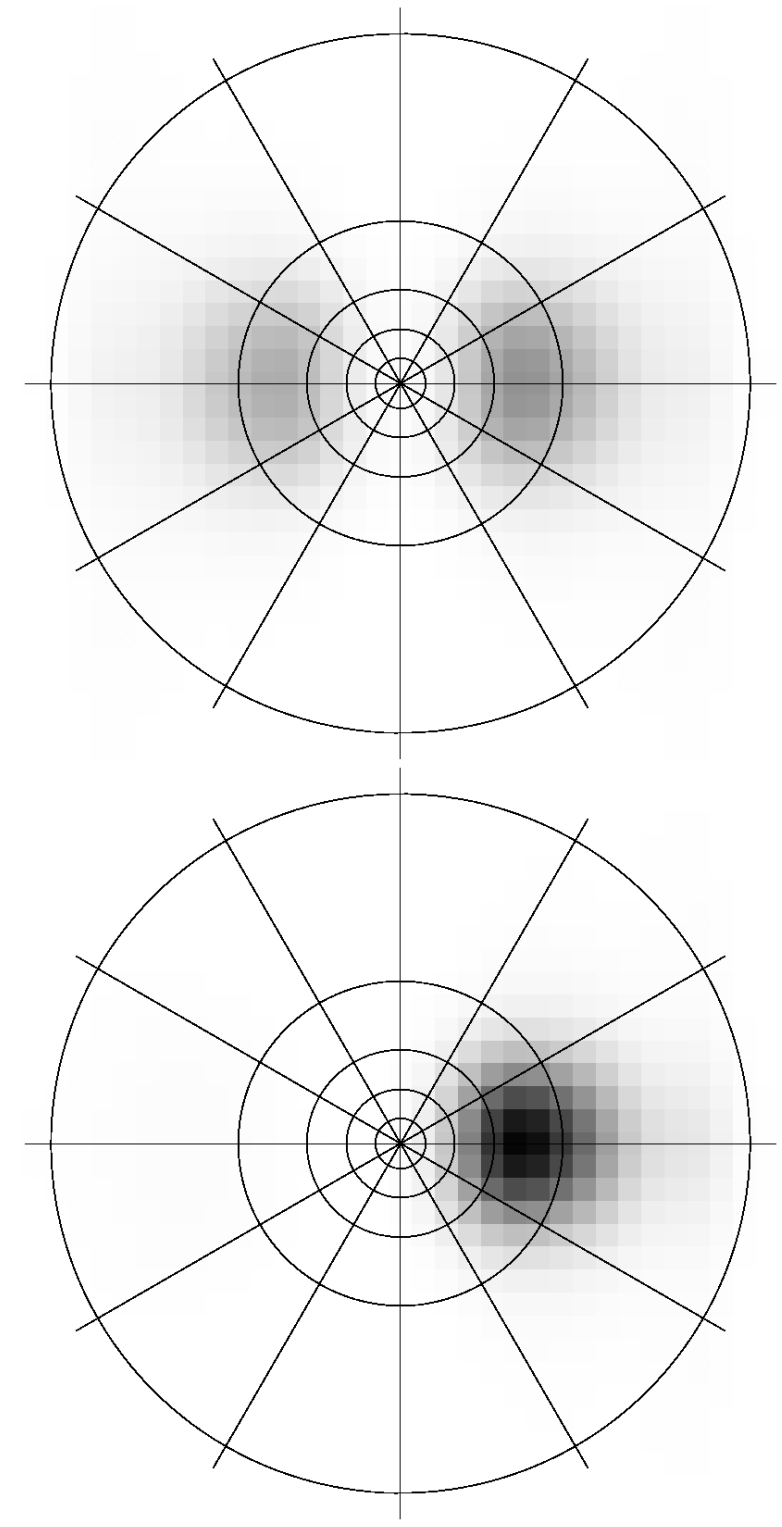

Fig. 2: Examples of probability distributions on the orientation of a plane. The polar angle is the tilt of the plane, the radius is the tangent of the slant angle, and the color stands for the probability. A darker color represents a higher probability. The peaks represent the most likely percepts, with the integral of the probability around a peak corresponding to the probability of the associated percept. The top panel shows a result with a high rate of depth reversals and the lower panel displays a low reversal rate.

Wexler et al. 2001a,b). An optic flow field such as the one shown in figure 1a leads, in the immobile observer, to total ambiguity between the solutions shown in figure $1 \mathrm{~b}$, and therefore a depth reversal rate of up to $50 \%$ for a small field of view. In the moving observer (figure 1c), on the other hand, the ambiguity is lifted in favor of the solution that is most stationary in an observerindependent reference frame (the left solution in figure 1c). 


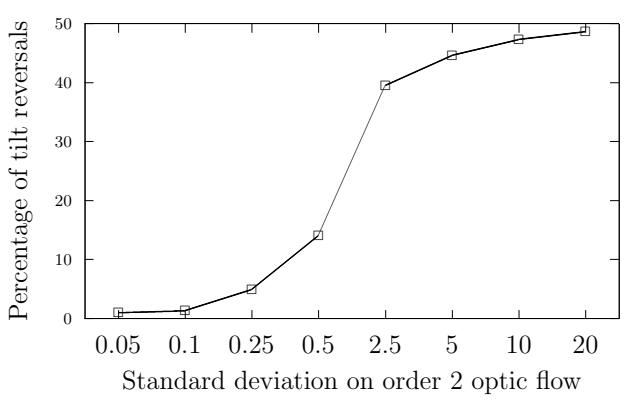

Fig. 3: Influence of the uncertainty of second-order optic flow on the reversal rate in the Bayesian model. A small field of vision leads to a greater uncertainty, and hence to more reversals.

The experimental data used as a reference is taken from van Boxtel et al. (2003), in which the perception of the same optic flow is compared in active and immobile conditions (figure 4), in a small field of view. The experimental results clearly reveal a bimodal distribution of tilt perception when the subject is immobile. There are two preferred responses around $0^{\circ}$, corresponding to the simulated plane, and $180^{\circ}$, corresponding to the depthreversed plane. In the active condition, the same optic flow is produced by the subject's displacement in front of an immobile plane. In this case, the depth-reversed plane is rarely reported, leading to a dominant peak in the distribution around $0^{\circ}$.
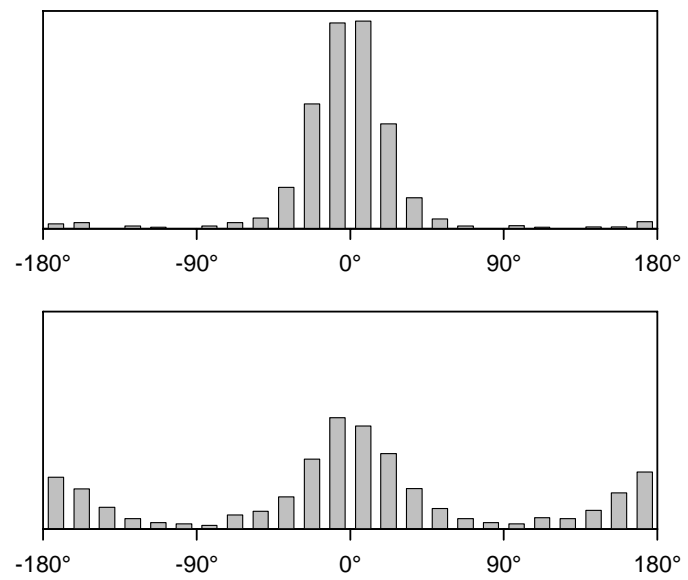

Fig. 4: Distributions of error in tilt angle for both active (top) and immobile (bottom) conditions, by van Boxtel et al. (2003). The results show depth reversals in the immobile condition and its almost complete disappearance in the active condition.

Figure 5 shows the results of our model in the same two conditions. We notice that the bimodality in the immobile condition is similar to the experimental results, and the decrease of reversals in the active condition. In the Bayesian model, the bimodality shown above is derived from the symmetry of the first-order optic
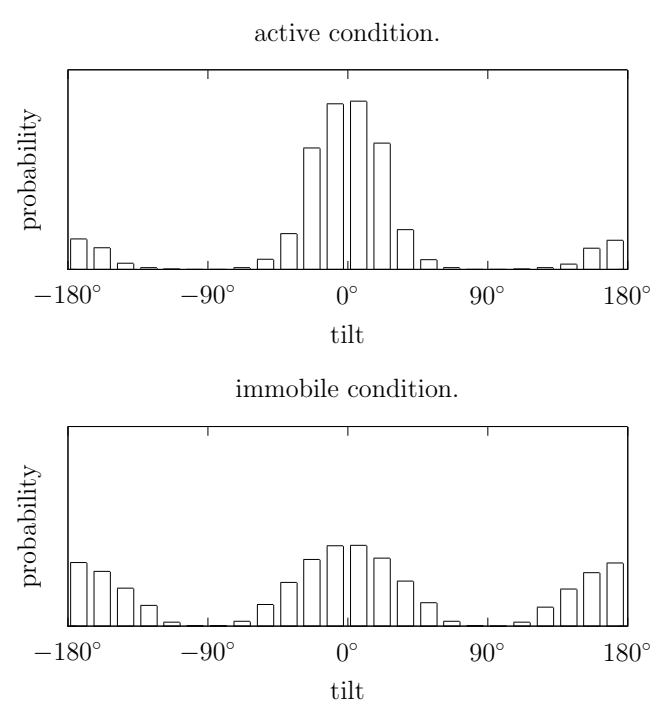

Fig. 5: Probability distributions of tilt errors in active and immobile conditions. As in the experimental results shown in figure 4, the ambiguity drastically diminishes in the active condition.

flow mentioned above. Furthermore, the difference between active and immobile conditions can be accounted for only by the conditional distribution on motion in an observer-independent reference frame. This distribution is the expression of the stationarity assumption in our model. In the immobile condition, the simulated and depth-reversed planes have the same speed, as depicted in figure 1(b); only the direction of motion changes. In the active condition, however, the simulated plane is stationary in an observer-independent reference frame, whereas the depth-reversed plane has high velocity $1(\mathrm{c})$. Therefore, the stationarity assumption, as implemented in the model, insures that the reversed plane is less probable, because it corresponds to a higher velocity in an observer-independent reference frame.

\subsection{Ambiguity between slant and speed}

The slant of a plane (the angle between the normal of the surface and the direction of gaze) is difficult to extract from optic flow. Indeed, the rotation around an axis lying in the fronto-parallel plane is entangled with surface slant. Starting from a given slant and motion configuration, simultaneously increasing slant and decreasing motion leads to approximately the same optic flow.

The experimental data we consider are taken from Domini and Caudek (1999). The experimental conditions involve a static monocular observer. The stimulus consists of a plane rotating along a fronto-parallel axis. The observer is asked to make a judgement about the slant of the plane. The planes can have two different slants and two different angular velocities. The relationship between the chosen slants is such that the tangent of the 
second slant is twice that of the first. The same holds for velocity, where the second is twice that of the first.

The experimental results, by Domini and Caudek (1999), are shown in table 4. The columns on the left show the evolution of the perception of the tangent of the slant angle while changing the values of angular speed or the simulated slant. These data show that the slant of the plane is hardly recovered as an independent variable, arguing against a veridical (Euclidean, review by Domini and Caudek (2003)) analysis of optic flow by human observers. Moreover, the perceived slant for small simulated slant and high angular speed is very close to the one perceived in the case of large simulated slant and low speed. Finally, this experiment shows that increasing the simulated slant or increasing the angular speed yields the same increase in perceived slant (around 23\% each time).

The right columns of table 4 show the predictions of our model in the same experimental conditions. Our model shows the slant/speed ambiguity found in the experimental results. In particular, the perceived slant for small slant with high angular speed is very close to the perceived slant for large slant with low angular speed. These results also show an increase in slant perception with increasing slant or speed. As in the experimental data, this increase is roughly the same (50 to 60\%) in both conditions, although greater than in the experimental data.

The perceived slant comes from a trade-off between our prior over the orientation (tilt and slant) of the plane and the distribution over the relative motion from the stationarity assumption (see Methods for details).

It is noted that the values of perceived slant for the model are slightly smaller than those of the experimental data, especially for a small simulated slant. We have chosen to provide the results of our model with a unique set of parameters for all the experiments of this section. These parameters are therefore a trade-off between the best parameters fitting each experiment.

\begin{tabular}{|c||c|r||r|r|}
\hline \multicolumn{1}{|c||}{} & \multicolumn{2}{c||}{ Experiment } & \multicolumn{2}{c|}{ Model } \\
\hline Angular speed & 0.25 & 0.5 & 0.25 & 0.5 \\
\hline \hline Small slant (1.5) & 1.13 & 1.29 & 0.66 & 1.00 \\
\hline Large slant (3) & 1.28 & 1.71 & 1.00 & 1.64 \\
\hline
\end{tabular}

Table 4: Mean perceived tangent of slant as a function of simulated slant tangent and angular speed for the experimental data (Domini and Caudek 1999) and the Bayesian model. Note the growth of perceived slant with increasing angular speed, and very similar perceived slant for large simulated slant/slow rotation and small simulated slant/fast rotation.

The slant/speed ambiguity results from ambiguities in first-order optic flow. Indeed, in both situations (small slant, high speed compared to large slant, low speed) the optic flow is the same up to the order one as shown in figure 1(e), and only the second-order optic flow could disambiguate the stimulus. These results confirm the low weighing of the second-order components of optic flow in a small field of view. This low weighing is due to the uncertainty attached to the distribution over the secondorder optic flow.

First-order optic flow depends on a parameter called def, the product of the tangent of the slant and angular speed (Domini and Caudek 2003). ${ }^{3}$ Therefore slant and speed cannot be recovered individually from first-order optic flow. Domini and Caudek (2003) propose a maximum-likelihood model to account for their psychophysical results. With a small size of field, in the absence of self-motion and translation, and disregarding second-order optic flow, the likelihood of our Bayesian model reduces to the Gaussian $P\left(\boldsymbol{\Phi}^{1} \mid \boldsymbol{\Omega} \Theta\right)$. The norm of first-order optic flow in this case is $\sqrt{\omega_{X}^{2}+\omega_{Y}^{2}} \sqrt{\chi^{2}+v^{2}}=|\boldsymbol{\Omega}| \tan \sigma$. Their model is thus a special case of our Bayesian model.

\subsection{Ambiguity of translation in depth}

Another symmetry or ambiguity of first-order optic flow is shown in figure 6. A rotation in depth generates the same (first-order) optic flow as a translation in depth together with a different rotation in depth, around an axis that differs by $90^{\circ}$ from the original rotation. It has been found (Wexler et al. 2001a; Wexler 2003) that the two solutions are perceived with different frequencies, depending on the observer's movement and the origin of depth translation, taht is, if the observer moves toward the surface, or if the surface moves toward the observer (see figure 6 ). These results can be summarized by stating that there is a strong bias toward perceiving the solution that minimizes motion in an observer-independent reference frame. Thus, these results provide further support for the stationarity assumption. However, the observer's percepts are also, by and large, in agreement with the rigidity assumption. Therefore, they provide a useful testing ground for our model, which incorporates both the stationarity and rigidity assumptions.

In the psychophysical studies, two conditions are tested: in the active condition, the observer moves his head in depth; in the immobile condition, the observer remains still but receives the same optic flow as in a previous active trial (Wexler et al. 2001a; Wexler 2003). ${ }^{4}$ In the active condition, the optic flow is generated by a plane rotating in depth, where the distance to the observer is fixed (the plane's center therefore undergoes

\footnotetext{
${ }^{3}$ Projected on vertical and transversal axes, def is $\chi \omega_{y}$, $v \omega_{y}, \chi \omega_{x}, v \omega_{x}$ in the equations shown in the appendix.

4 Other conditions, involving conflict between the observer's motor command and self-motion, were also tested (Wexler 2003), and found to lead to different response distributions.
} 


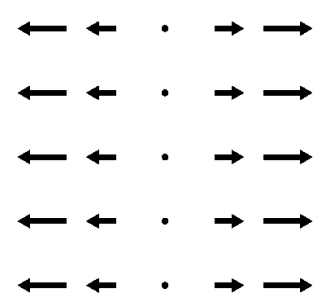

(a)

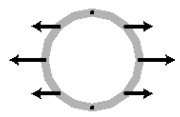

(b)

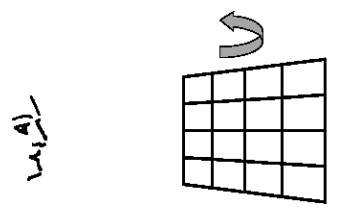

(c)

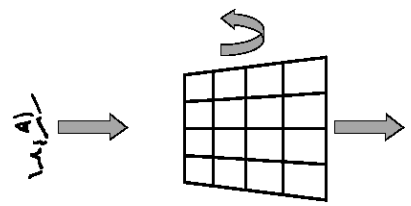

(d)

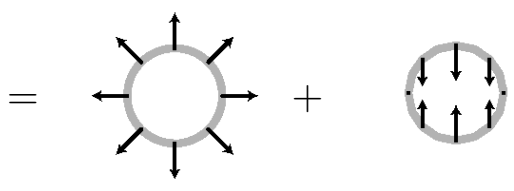

$\left(b^{\prime}\right)$

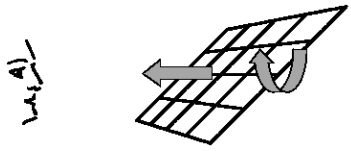

$\left(c^{\prime}\right)$

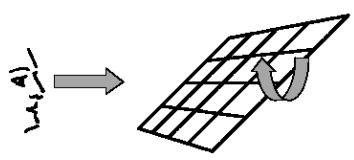

$\left(d^{\prime}\right)$

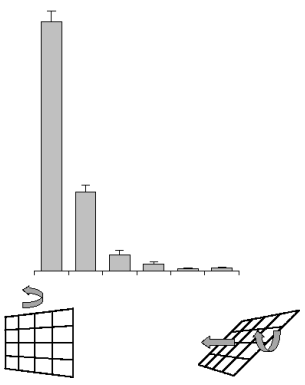

$\left(c^{\prime \prime}\right)$

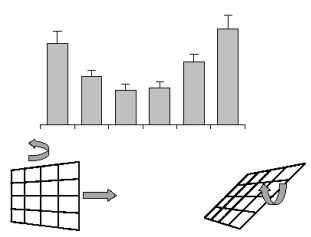

$\left(d^{\prime \prime}\right)$

Fig. 6: Illustration of the effect of head motion on the perception of 3-D structures (Wexler et al. 2001a; Wexler 2003). (a) An ambiguous 2D optic flow field that can have different 3-D interpretations, discovered by J. Droulez (cf fig. 1(a)). The arrows represent the motion of projections of points in 3-D space on the retina. It is fairly easy to see that the 3-D configuration shown in (c) will generate this flow. However, the configuration shown in ( $\left.\mathrm{c}^{\prime}\right)$ can also generate the flow in (a), and the reason for this is shown in $(b)$ and $\left(b^{\prime}\right)$ : if the amplitudes of the translation and rotation in $\left(\mathrm{c}^{\prime}\right)$ are adjusted correctly, the rotation can exactly cancel the expansion flow from the depth translation in one of two dimensions. The planes in (c) and ( $\left.\mathrm{c}^{\prime}\right)$ have the same slant and angular speed, but different tilts and they rotate about different axes. $(d),\left(d^{\prime}\right)$ Because optic flow depends only on the relative motion between object and observer, the same ambiguity holds for an observer moving forward and experiencing the optic flow in (a). If the observer's speed is equal-and-opposite to the translation in $\left(\mathrm{c}^{\prime}\right)$, the stationarity of the solutions is reversed with respect to $(c)$ and $\left(c^{\prime}\right)$ : it is now the center of $\left(d^{\prime}\right)$ that is stationary in space, while $(d)$ translates at the same speed as the observer. $\left(\mathrm{c}^{\prime \prime}\right),\left(\mathrm{d}^{\prime \prime}\right)$ Data by Wexler (2003) show the frequencies of the perceived solutions for stationary $\left(\mathrm{c}^{\prime \prime}\right)$ and moving $\left(\mathrm{d}^{\prime \prime}\right)$ observers, with the bars on the left corresponding to solutions (c) and (d), and the bars on the right to solutions $\left(c^{\prime}\right)$ and $\left(d^{\prime}\right)$. Although optic flow is the same in the two cases, perceptions of 3-D structure are very different, showing the effect of the observer's action.

depth translation as well). Therefore, in the active condition $6(\mathrm{~d})$, the rigidity assumption favours the simulated plane, while the stationarity assumption favours the alternative solution. ${ }^{5}$ In the immobile condition, on the

\footnotetext{
5 The reason why the rigidity assumption favours the simulated plane rather than the alternative solution is that the symmetry of figure 6 only holds for first-order optic flow. The
}

other hand, both the rigidity and stationarity assumptions favour the simulated plane.

The experimental results are presented in figure $6\left(\mathrm{c}^{\prime \prime}\right),\left(\mathrm{d}^{\prime \prime}\right)$ and in table 5 as the fraction of trials in which the observers perceive the alternative, nonrigid

second-order terms break the symmetry, and lead to nonrigidity of the alternative solution. 


\begin{tabular}{|c|r|r|}
\hline Condition & Experiment & Model \\
\hline Active & $54.3 \%$ & $79.6 \%$ \\
\hline Immobile & $3.6 \%$ & $17.8 \%$ \\
\hline
\end{tabular}

Table 5: Rate of alternative, non-rigid responses for the ambiguous depth-translation stimulus. Experimental results by Wexler (2003) (which do not explicitly state the immobile results). The higher rate in the active condition than in the passive condition is due to the stationarity assumption. In the immobile condition, both the stationarity and rigidity assumptions favor the same percept.

plane. Recall that optic flow is the same in the active and immobile conditions; only the observers' differs. Providing that only first-order optic flow components are available, the rigidity assumption alone would predict equally low rates for the alternative solution in the two conditions, whereas stationarity alone would result in a rate close to $100 \%$ in the active condition and a low rate in the immobile condition. Second-order optic flow components, if available, would decrease the rate for the alternative nonrigid solution.

As explained above, the discrepancy between the actual values of the experimental results and the model are due to the unique parameter set used for all six experiments. More precisely, different groups of participants already exhibit differences in their results. See, for instance, figure $6\left(\mathrm{c}^{\prime \prime}\right)$ and the histogram at the bottom left in figure 8. Both correspond to the same conditions but the results are numerically different. Priors in our model can be adjusted to better fit some results at the expense of other experiments.

Because our model implements both the rigidity and stationarity assumptions, they are in competition when the most rigid and most stationary objects do not match. In this experiment, such a mismatch happens in the active condition. The model deals with this kind of contradiction in a way that is similar to Bayesian fusion (Lebeltel et al. 2004). Other instances of Bayesian fusion are exemplified in the literature (Landy et al. 1995; Ernst and Banks 2002). The uncertainty, as quantified by the probability distributions, will ensure the optimal balance between the rigidity and stationarity assumptions.

\subsection{The effect of shear on SFM}

Another point we tested with the Bayesian model is the effect of the shear component of optic flow on SFM performance. The shear angle is the absolute difference between the tilt angle and the direction of the frontal translation. It is called "winding angle" by Cornilleau-Pérès et al. (2002). Psychophysical studies have found that SFM performance in immobile human observers (namely, judgement of tilt) deteriorates drastically as shear increases (Cornilleau-Pérès et al. 2002), but that this deterioration is much less drastic in active observers gen-

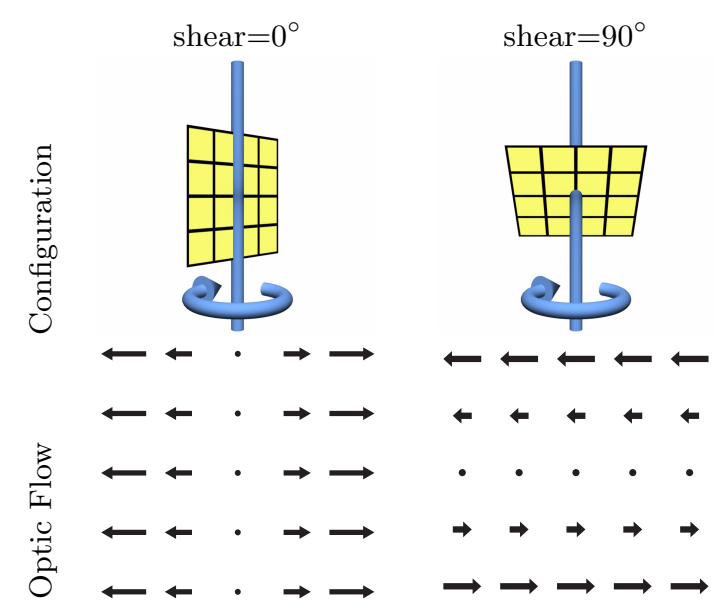

Fig. 7: Illustration of shear in optic flow. Shear can be parametrized by the shear angle, defined as $90^{\circ}$ minus the absolute value of the difference between tilt and axis angles. Configurations corresponding to two values of shear angle are shown; $0^{\circ}$ (minimum shear) and $90^{\circ}$ (maximum). The bottom row shows the optic flow resulting from each configuration.

erating optic flow through their own head movements (van Boxtel et al. 2003). Examples of minimal and maximal shear in optic flow are shown in figure 7. Shear can be parametrized by the shear angle (which takes values between $0^{\circ}$, corresponding to no shear, and $90^{\circ}$, corresponding to maximal shear).

We compared model results to experimental findings by van Boxtel et al. (2003). The experiment involves a monocular observer who is either immobile, or moving in a direction perpendicular to direction gaze (active condition). In the two conditions, the observer receives the same optic flow. In the active condition, the simulated plane is stationary in an observer-independent reference frame. In the immobile condition, the plane rotates about an axis in the fronto-parallel plane. The observer's task is to report the plane's orientation by aligning a probe so that it appears parallel to the plane.

Figure 8 shows the distribution of absolute tilt errors from the experimental results (van Boxtel et al. 2003), in both active and immobile conditions, for minimal and maximal shear. We can see that mean errors increase with increasing shear. However, this effect is much stronger in the immobile condition (where response is almost at chance level for highest shear) than in the active condition.

Figure 9 shows the distribution of absolute tilt errors for the same conditions as given by the model. The variation of the precision between low and high shear is similar to the experimental results.

In the model, the main factor inducing the shear effect is the relative weight of the rotation prior and the translation prior. Indeed, for a small shear, the absolute motion that satisfies the first-order optic flow equations for a large tilt error is composed of a rotation and a 
Shear 0

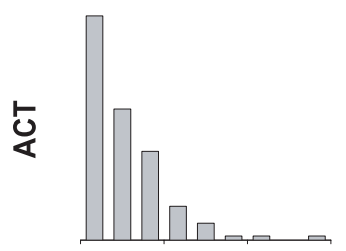

Shear 90

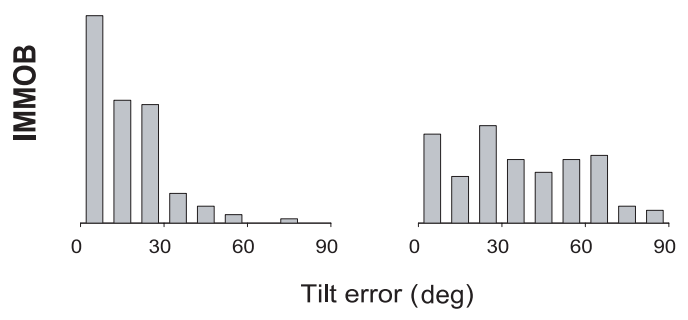

Fig. 8: Tilt error for both active and immobile conditions and shear $0^{\circ}$ and $90^{\circ}$, by van Boxtel et al. (2003). Tilt reversals (much more common in the immobile condition, see figure 4) were corrected by using the opposite tilt from the one reported in calculating errors, when an reversal occurred; thus, absolute tilt error runs between $0^{\circ}$ and $90^{\circ}$.
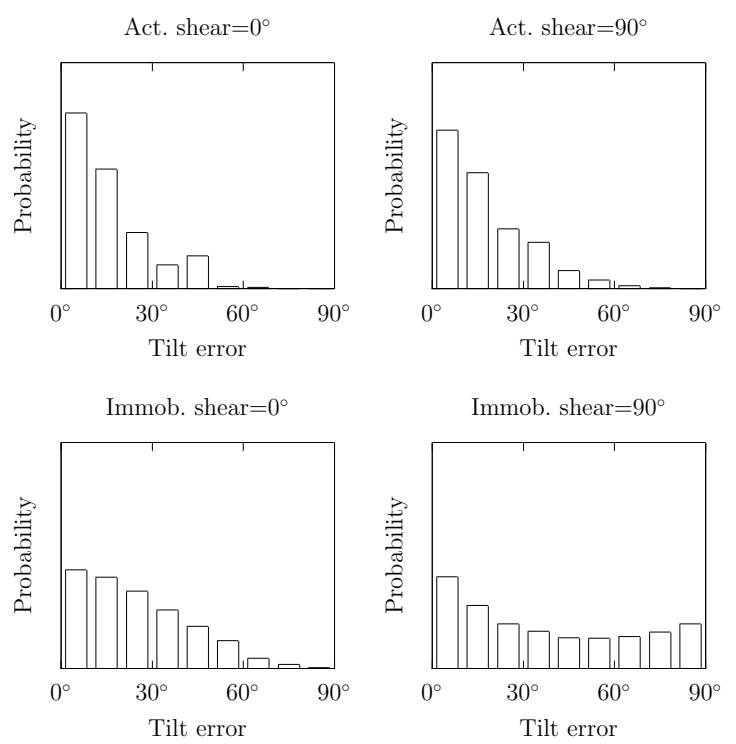

Fig. 9: The effect of shear and observer motion on tilt error, as predicted by the Bayesian model. As in the experimental results (figure 8), the mean tilt error is greater for a $90^{\circ}$ shear than for $0^{\circ}$ and this effect is greater for an immobile observer than an active one.

translation. For a high shear, a large error corresponds to an absolute motion composed of two rotations with the same velocity. The stationarity assumptions states that both the translation and the rotation components of the absolute motion are probably small. However, the constraint on the translation component should be stronger than on the rotation component in order to reduce the dispersion of tilt error for small shear. The strength of the shear effect depends on the relative strength of the stationarity constraints on translation and rotation components.

\subsection{Influence of eye movements on 3-D vision}

Using a sinusoidally curved surface that underwent lateral translation while being pursued with the eyes by the subject, Naji and Freeman (2004) found few depth reversals. However, when the same optic flow was presented without pursuit (i.e., with the translation substracted), depth reversals were prevalent. We simulated a very similar experiment, with the only difference being that we used a planar rather than a curved surface. Because planes can undergo depth reversals in the same way as curved surfaces, the main effect found by Naji and Freeman, or something very close it, can be simulated within the framework of our model.

As can be seen in figure 1d (analogous to condition C by Naji and Freeman (2004)), depth reversals can take place in the pursuit condition. Both solutions undergo the same translation, and one of the solutions additionally undergoes a rotation. In the fixation condition (analogous to condition B by Naji and Freeman (2004)), the same optic flow leads to two solutions undergoing equaland-opposite rotations, as shown in figure 1b. Finally, Naji and Freeman (2004) have a third condition (A) where the object translates as in condition $\mathrm{C}$, but in which the observers were required to fixate on a stationary point rather than pursue the object.

The rate of depth reversals is calculated from subjects' responses in a depth-order task. Figure 10 shows the experimental results of these three conditions. The graphs show the estimation of the phase with respect to the amplitude of the stimulus. The phase is the analog of the orientation of the plane in figures $1(\mathrm{~d})$ and (b), whereas the amplitude stands for the slant of the plane (negative slant being a reversal). We notice that translation ( $\mathrm{A}$ and $\mathrm{C}$ ) allows for the disambiguation of the stimulus, whereas rotation exhibits a symmetric behavior. We notice that the perception is more precise in the pursuit condition $(\mathrm{C})$ than the immobile condition $(\mathrm{A})$.

In comparison, figure 11 shows the results of the Bayesian model in the transposed conditions. We can see the major properties are reproduced, in particular the broader uncertainty in condition A compared to condition $\mathrm{C}$, as well as the ambiguity in condition $\mathrm{B}$.

Until now, subjectives responses were limited to the plane orientation. An additional element has to be included in the model in order to account for the 'topfar' responses. This decision was made using a simple Bayesian program. As can be seen in conditions A and $\mathrm{C}$ in figure 10, the observers exhibited some preference toward a 'top-far' perception. This preference is included as a prior in Bayesian post-processing. However, it is to be noted that observers seem to have a preference for a 'top-near' perception in condition B. 
(A)

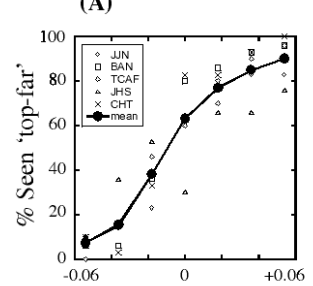

(B)

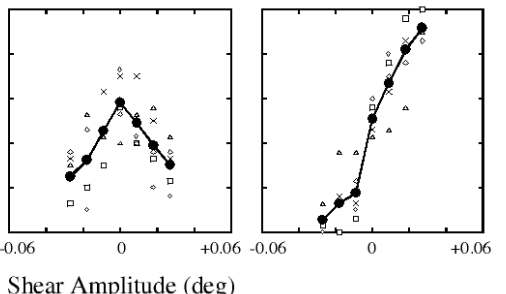

Shear Amplitude (deg)

(C)
Fig. 10: Rate of 'top-far' perception with respect to the strength of the stimulus (Naji and Freeman 2004). Condition A corresponds to a translating object without eye pursuit; condition $\mathrm{B}$ to a rotating object and condition $\mathrm{C}$ to a translating object with pursuit. Conditions $\mathrm{A}$ and $\mathrm{B}$ show that translation allows for a disambiguation, contrary to passive rotation. Furthermore, the comparison of conditions $\mathrm{A}$ and $\mathrm{C}$ shows that pursuit of the object leads to better perception.
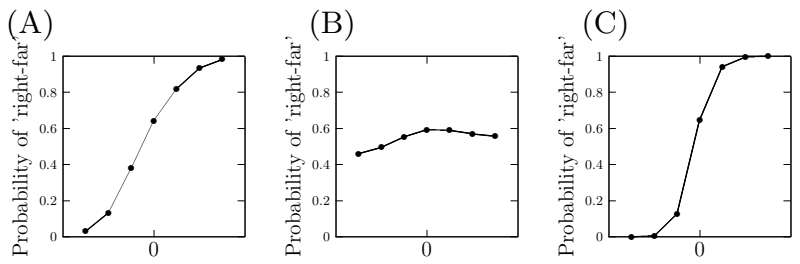

Fig. 11: Results from the model. As for the experimental results, conditions $\mathrm{A}$ and $\mathrm{C}$ allow for disambiguation of the stimulus, and condition $\mathrm{C}$ is less uncertain than condition $\mathrm{A}$.

The results in condition $\mathrm{B}$ are the same as those in the immobile condition above. The small asymmetry of both top and bottom curves comes from the second-order optic flow that induces a reversal rate strictly less than $50 \%$.

The difference between the model results in conditions $\mathrm{A}$ and $\mathrm{C}$ comes from the stationarity of the reverse percepts. In condition $\mathrm{C}$, the reverse percept undergoes a greater rotation than in condition $\mathrm{A}$. Therefore, the stationarity assumption assigns it a smaller probability, hence yielding a smaller reversal rate.

\section{Discussion}

\subsection{Probabilistic expression of assumptions}

A Bayesian model infers the logical consequences of a given set of assumptions with some observations. The inference can occur as soon as a joint probability distribution is defined. Therefore, the modeler has to express the assumptions in a Bayesian way.

Each choice in specification is an assumption. As there are multiple steps in the specification of a joint probability distribution, there are multiple levels of expression. The first choice is the variables and their domain. The variables absent at this step cannot have a meaningful influence in the model. Then, the joint distribution is decomposed into a product of factors using conditional independencies. The conditional independencies express a lack of relationship between variables and therefore simplify the inference. The last level of expression of assumptions is in the specification of each distribution and its parameters appearing into the decomposition.

Each choice is a reduction in the degrees of freedom of the joint distribution. The more drastic restrictions are in the choice of the variables and their domain while the less important are in the choice of the parameters of the distribution. Any reduction can be postponed to a later stage but the earlier it is done, the more the inference can take advantage of it to simplify the computations.

\subsection{Choices in our Bayesian model}

The issue of specification is therefore to express our assumptions in terms of choice of variable, simplification of the joint distribution using conditional independencies, and choice of parametric forms and parameters.

The first main assumption is that of rigidity, which states that the optic flow more likely to be observed is generated by a plane in relative motion. The parametric space of the optic flow is derived from this assumption. The optic flow is defined by eight parameters. While sufficient in the case of a plane, the optic flow is, in general, more complicated. This means that other eventual components are not relevant variables in our model, and are therefore ignored. It could be interesting to investigate an eventual effect of these components in the human perception of a plane. As far as the model is concerned, such investigation can be studied with additional components in the optic flow variable. Rigidity is also preeminent in the choice of the parametric form of the probability distribution over optic flow, given relative motion, position of the plane, and the conditions of observation. We fixed this as a Gaussian distribution. However, it would be possible to evaluate this choice of distribution by measuring evolution of performance with respect to some additional noise in the stimulus and comparing it to the predicted evolution of the model.

The other main assumption of our model is that of stationarity, which states that the motion of the plane is more likely to be small. The variables chosen to describe the motion are the translation and rotation components along the three axes, according to the experiments chosen as references. This is restrictive in the sense that it does not take into account eventual accelerations and even more complex trajectory. Most reported studies deal with uniform motion, however, investigation of the influence of accelerations in the perception of structure could benefit from the model. The model can be adapted to implement and predict the results of different hypotheses to be compared to experimental results. 
The parameters are the last elements of choice in the model. We obtained the results presented above with a single set of parameters. Each experimental result gives information on the exact effect highlighted by the experiment on some parameters. However, the optimal parameters for each experiment are different; therefore, the final set of parameters chosen results from a trade-off between all the experiments.

\subsection{Model results}

The results of the model display some discrepancies with the results of the experiments. For example, for the first experiment described, the reversal rate of the model in a small field is $44.6 \%$ compared with $48.8 \%$ in the experiment (Cornilleau-Pérès et al. 2002). There are two main reasons for this difference. First, the Bayesian model is a model of an observer. It is not specifically designed to reproduce mean results across observers. Nevertheless, the results of our model are less than the variability reported between observers (in this case, the minimum reversal rate reported by Cornilleau-Pérès et al. (2002) is around $38 \%)$. As explained above, the set of parameters is the same across all the results of our model. However, there are variations in the precise experimental conditions between the different teams responsible for the measured results. For instance, the rate of reversal measured in a small field of view for an immobile observer by van Boxtel et al. (2003) is $35 \%$, compared with $48.8 \%$ measured by Cornilleau-Pérès et al. (2002). This can be explained by differences in the protocol that are not taken into account as relevant variables in the Bayesian model. Therefore, as a general rule, the parameters we chose for the Bayesian model are a trade-off between all the results. This way, the results of the model cannot precisely match those of the experimental results.

The Bayesian model not only accounts for previously reported results but can also be used to make predictions and eventually propose new experiments. For example, we propose the investigation of the relative influence of stationarity and rigidity in large fields of view. In this case, in an experimental setup similar to that of Wexler (2003), our model predicts that rigidity will be of greater importance in the perception of second-order optic flow through a diminution of standard deviation on these components.

Another prediction of the Bayesian model involves the shear effect. In our model, this effect is accounted for by relative weight between rotation and translation components in a small field of vision. Our model predicts a reduced shear effect in large fields of vision, and this has been found in human observers (Cornilleau-Pérès et al. 2002).

Acknowledgements This work was supported by the European Project BIBA IST-2001-32115.

\section{A Optic flow equations}

Let $\mathcal{P}$ be the object plane, $(\chi, v)$ its depth gradients, $\widetilde{M}$ of coordinates $(\tilde{x}, \tilde{y}, \tilde{z})$ a point of this plane in the 3-D reference frame, and $M$ of coordinates $(x, y)$ its projection in the image plane. The equation of the plane is:

$$
\tilde{x} \chi+\tilde{y} v-\tilde{z}=0 .
$$

We have the slant of the plane $\sigma=\arctan \sqrt{\chi^{2}+v^{2}}$ and the tilt $\tau=\arctan \frac{v}{\chi}$ :

Let $P$ be the projection of a 3-D point in the image:

$$
P: \quad\left(\begin{array}{l}
\tilde{x} \\
\tilde{y} \\
\tilde{z}
\end{array}\right) \mapsto\left(\begin{array}{l}
x=\frac{\tilde{x}}{1 \tilde{z}} \\
y=\frac{\tilde{y}}{1-\tilde{z}}
\end{array}\right) .
$$

Let $\mathbf{t}=\left(t_{x}, t_{y}, t_{z}\right)$ and $\boldsymbol{\omega}=\left(\omega_{x}, \omega_{y}, \omega_{z}\right)$ respectively be the translation and rotation vector of the object plane.

Considering the points as functions of time, we can write:

$$
M(t)=P \circ \widetilde{M}(t)
$$

Optic flow is the displacement of the points in the image:

$$
\begin{aligned}
\phi & =\frac{d M}{d t} \\
\phi & =\frac{d P}{d \widetilde{M}}(\widetilde{M}) \times \frac{d \widetilde{M}}{d t} .
\end{aligned}
$$

$\frac{d P}{d \widetilde{M}}$ is the Jacobian of $P$ :

$$
\begin{aligned}
& \frac{d P}{d \widetilde{M}}(\widetilde{M})=\left(\begin{array}{lll}
\frac{\partial x}{\partial \tilde{x}} & \frac{\partial x}{\partial \tilde{y}} & \frac{\partial x}{\partial \tilde{z}} \\
\frac{\partial y}{\partial \tilde{x}} & \frac{\partial y}{\partial \tilde{y}} & \frac{\partial y}{\partial \tilde{z}}
\end{array}\right) \\
& \frac{d P}{d \widetilde{M}}(\widetilde{M})=\left(\begin{array}{ccc}
\frac{1}{1-\tilde{z}} & 0 & \frac{\tilde{x}}{(1-\tilde{z})^{2}} \\
0 & \frac{1}{1-\tilde{z}} & \frac{\tilde{y}}{(1-\tilde{z})^{2}}
\end{array}\right)
\end{aligned}
$$

The plane $\mathcal{P}$ undergoes translation $\mathbf{t}$ and rotation $\boldsymbol{\omega}$. Therefore the motion $\frac{d \widetilde{M}}{d t}$ of $\widetilde{M}$ is:

$$
\begin{aligned}
\frac{d \widetilde{M}}{d t} & =\mathbf{t}+\boldsymbol{\omega} \wedge \widetilde{M} \\
& =\left(\begin{array}{c}
t_{x} \\
t_{y} \\
t_{z}
\end{array}\right)+\left(\begin{array}{c}
\omega_{x} \\
\omega_{y} \\
\omega_{z}
\end{array}\right) \wedge\left(\begin{array}{c}
\tilde{x} \\
\tilde{y} \\
\chi \tilde{x}+v \tilde{y}
\end{array}\right) \\
\frac{d \widetilde{M}}{d t} & =\left(\begin{array}{c}
t_{x}+\chi \omega_{y} \tilde{x}+\left(v \omega_{y}-\omega_{z}\right) \tilde{y} \\
t_{y}+\left(\omega_{z}-\chi \omega_{x}\right) \tilde{x}-v \omega_{x} \tilde{y} \\
t_{z}+\omega_{x} \tilde{y}-\omega_{y} \tilde{x}
\end{array}\right)
\end{aligned}
$$

Substituting 14 and 15 in equation 13, we get:

$$
\begin{aligned}
\phi & =\frac{d P}{d \widetilde{M}}(\widetilde{M}) \times \frac{d \widetilde{M}}{d t} \\
& =\left(\begin{array}{ccc}
\frac{1}{1-\tilde{z}} & 0 & \frac{\tilde{x}}{(1-\tilde{z})^{2}} \\
0 & \frac{1}{1-\tilde{z}} \frac{\tilde{y}}{(1-\tilde{z})^{2}}
\end{array}\right) \times\left(\begin{array}{c}
t_{x}+\chi \omega_{y} \tilde{x}+\left(v \omega_{y}-\omega_{z}\right) \tilde{y} \\
t_{y}+\left(\omega_{z}-\chi \omega_{x}\right) \tilde{x}-v \omega_{x} \tilde{y} \\
t_{z}+\omega_{x} \tilde{y}-\omega_{y} \tilde{x}
\end{array}\right) \\
\phi & =\left(\begin{array}{c}
\frac{t_{x}+\chi \omega_{y} \tilde{x}+\left(v \omega_{y}-\omega_{z}\right) \tilde{y}}{1-\tilde{z}}+\frac{\tilde{x}}{1-\tilde{z}} \times \frac{t_{z}+\omega_{x} \tilde{y}-\omega_{y} \tilde{x}}{1-\tilde{z}} \\
\frac{t_{y}+\left(\omega_{z}-\chi \omega_{x}\right) \tilde{x}-v \omega_{x} \tilde{y}}{1-\tilde{z}}+\frac{\tilde{y}}{1-\tilde{z}} \times \frac{t_{z}+\omega_{x} \tilde{y}-\omega_{y} \tilde{x}}{1-\tilde{z}}
\end{array}\right)
\end{aligned}
$$


By definition of $P$ (equation 10), $\frac{\tilde{x}}{1-\tilde{z}}=x, \frac{\tilde{y}}{1-\tilde{z}}=$ $y$ and $\frac{1}{1-\tilde{z}}=1+\chi x+v y$. We can finally rewrite the equation 16 to get the equations 17 of the optic flow of a plane:

$\phi=\left(\begin{array}{l}\frac{t_{x}+\chi \omega_{y} \tilde{x}+\left(v \omega_{y}-\omega_{z}\right) \tilde{y}}{1-\tilde{z}}+\frac{\tilde{x}}{1-\tilde{z}} \times \frac{t_{z}+\omega_{x} \tilde{y}-\omega_{y} \tilde{x}}{1-\tilde{z}} \\ \frac{t_{y}+\left(\omega_{z}-\chi \omega_{x}\right) \tilde{x}-v \omega_{x} \tilde{y}}{1-\tilde{z}}+\frac{\tilde{y}}{1-\tilde{z}} \times \frac{t_{z}+\omega_{x} \tilde{y}-\omega_{y} \tilde{x}}{1-\tilde{z}}\end{array}\right)$

$\boldsymbol{\phi}=\left(\begin{array}{c}t_{x}+x\left[t_{z}+\chi\left(t_{x}+\omega_{y}\right)\right]+y\left[-\omega_{z}+v\left(t_{x}+\omega_{y}\right)\right] \\ +x^{2}\left(\chi t_{z}-\omega_{y}\right)+x y\left(v t_{z}+\omega_{x}\right) \\ t_{y}+x\left[\omega_{z}+\chi\left(t_{y}-\omega_{x}\right)\right]+y\left[t_{z}+v\left(t_{y}-\omega_{x}\right)\right] \\ +x y\left(\chi t_{z}-\omega_{y}\right)+y^{2}\left(v t_{z}+\omega_{x}\right)\end{array}\right)$

$\phi=\phi^{0}+\phi^{1} \cdot{ }^{t}(x, y)+{ }^{t}(x, y) .^{t} \phi^{2} \cdot{ }^{t}(x, y)$

with:

$$
\begin{aligned}
\phi^{0} & =\left(\begin{array}{l}
t_{x} \\
t_{y}
\end{array}\right) \\
\phi^{1} & =\left(\begin{array}{cc}
t_{z}+\chi\left(t_{x}+\omega_{y}\right) & -\omega_{z}+v\left(t_{x}+\omega_{y}\right) \\
\omega_{z}+\chi\left(t_{y}-\omega_{x}\right) & t_{z}+v\left(t_{y}-\omega_{x}\right)
\end{array}\right) \\
\phi^{2} & =\left(\begin{array}{c}
\chi t_{z}-\omega_{y} \\
v t_{z}+\omega_{x}
\end{array}\right)
\end{aligned}
$$

\section{References}

V. Cornilleau-Pérès, M. Wexler, J. Droulez, E. Marin, C. Miège, and B. Bourdoncle. Visual perception of planar orientation: dominance of static depth cues over motion cues. Vision Research, 42:1403-1412, 2002.

T. Dijkstra, V. Cornilleau-Pérès, C. Gielen, and J. Droulez. Perception of three-dimensional shape from ego- and object-motion: comparison between small- and large-field stimuli. Vision Research, 35(4):453-462, 1995.

F. Domini and C. Caudek. Perceiving surface slant from deformation of optic flow. J Exp Psychol Hum Percept Perform., 25(2):426-44, 1999.

F. Domini and C. Caudek. 3-d structure perceived from dynamic information: a new theory. Trends in Cognitive Sciences, 7(10):444-9, 2003.

M. Ernst and M. Banks. Humans integrate visual and haptic information in a statistically optimal fashion. Nature, 415 (6870):429-33, 2002.

D. Kersten, P. Mamassian, and A. Yuille. Object perception as bayesian inference. annu Rev Psychol., 55:271-304, 2004.

J. Koenderik. Optic flow. Vision Research, 26(1):161-179, 1986.

M. Landy, L. Maloney, E. Johnston, and M. Young. Measurement and modeling of depth cue combination: in defense of weak fusion. Vision Research, 35:389-412, 1995.

O. Lebeltel, P. Bessière, J. Diard, and E. Mazer. Bayesian robot programming. Advanced Robotics, 16(1):49-79, $2004 . \quad$ URL http://emotion.inrialpes.fr/bibemotion/2004/LBDM04.

$H$. Longuet-Higgins. The visual ambiguity of a moving plane. Proceedings of the Royal Society of London (B, Biological Sciences), 223:165-175, 1984.

J. Mayhew and H. Longuet-Higgins. A computational model of binoculard depth perception. Nature, 297(5865):376-8, 1982.
J. Naji and T. Freeman. Perceiving depth order during pursuit eye movement. Vision Research, 44:3025-34, 2004.

B. Rogers and M. Graham. Motion parallax as an independent cue for depth perception. Perception, 8:125-134, 1979.

B. Rogers and S. Rogers. Visual and nonvisual information disambiguate surfaces specified by motion parallax. Perception and Psychophysics, 52:446-452, 1992.

J. Todd and P. Bressan. The perception of 3-dimensional affine structure from minimal apparent motion sequences. Perception and Psychophysics, 45(5):419-30, 1990.

J. Todd and J. Norman. The visual perception of smoothly curved surfaces from minimal apparent motion sequences. Perception and Psychophysics, 50(6):509-23, 1991.

S. Ullman. The interpretation of visual motion. Cambridge: MIT Press, 1979.

J. van Boxtel, M. Wexler, and J. Droulez. Perception of plane orientation from self-generated and passively observed optic flow. Journal of Vision, 3(5):318-332, 2003. URL http://journalof vision.org/3/5/1/.

H. von Helmholtz. Handbuch der Physiologischen Optik. Hamburg: Voss, 1867.

H. Wallach and D. O'Connell. The kinetic depth effect. Journal of Experimental Psychology, 45:205-217, 1953.

H. Wallach, J. Stanton, and D. Becker. The compensation for movement-produced changes in object orientation. Perception and Psychophysics, 15:339-343, 1974.

Y. Weiss, E. Simoncelli, and E. Adelson. Motion illusions as optimal percepts. Nature Neuroscience, 5(6):508-10, 2002.

M. Wexler. Voluntary head movement and allocentric perception of space. Psychological Science, 14:340-346, 2003.

M. Wexler, I. Lamouret, and J. Droulez. The stationarity hypothesis: an allocentric criterion in visual perception. Vision Research, 41:3023-3037, 2001a.

M. Wexler, F. Panerai, I. Lamouret, and J. Droulez. Selfmotion and the perception of stationary objects. Nature, 409:85-88, 2001b. 